Case Report

\title{
Management of Congenital Muscular Torticollis (Cmt) with a Multidisciplinary Approach
}

\author{
Anurag Gattani ${ }^{1}$, Vinaya Bhat ${ }^{2}$, Chethan Hegde ${ }^{3}$, Manoj Shetty ${ }^{4}$, Padmaraj J. Hegde $^{5}$, Ashish J. Rai ${ }^{6}$, \\ RajJaiswal ${ }^{7}$ \\ ${ }^{1}$ Former P.G. Student, ${ }^{2}$ Professor, ${ }^{3}$ Professor and Head, Dept. of Prosthodontics, ${ }^{4}$ Professor and Head, department of Oral \\ Implantology, ${ }^{5,6}$ Lecturer, ${ }^{7}$ P.G. Student, Dept. of Oral \& M axillofacial Surgery, A.B. Shetty M emorial Institute Of Dental \\ Sciences, M angalore.
}

*Corresponding Author : Vinaya Bhat, Professor, Dept. of Prosthodontics, A.B. Shetty M emorial Institute of Dental Sciences, Mangalore.

Received

: 05.03 .2018

Review Completed : 12.06.2018

Accepted

: 18.07.2018

Keywords : Congenital Muscular Torticollis (CMT), wry neck, Sternocleidomastoid muscle, bipolar surgery

\begin{tabular}{|c|}
\hline Access this article online \\
\hline Quick Response Code \\
\hline \\
\end{tabular}

\begin{abstract}
Congenital M uscular Torticollis (CMT) is a rare condition which is seen in childhood where there is difficulty in rotating the neck and the head is tilted to one side following the stiffness of the sternocleidomastoid muscle. When the patient reports for treatment during adulthood, a multidisciplinary approach involving surgical, prosthodontic and physiotherapy procedures are required. In this case report, the authors are describing successful management of an adult patient affected with CM T.
\end{abstract}

\section{Introduction}

Congenital Muscular Torticollis (CMT) (Wry neck) is the most common form of torticollis occurring in an infant and/or a young child. The characteristic head tilt seen in this condition is caused by abnormal contracture of the sternocleidomastoid muscle. With contracture of the muscle, the head tilts towards the involved side with the chin rotated towards the opposite shoulder. This also leads to a limitation of the head mobility in both rotation and lateral flexion and progressive degrees of neck contracture. ${ }^{1}$ Other causes of the wry neck include sequelae to inflammatory, ocular, neurologic or orthopedic diseases. ${ }^{2}$ This paper presents successful rehabilitation of a CMT patient with a multidisciplinary approach.

\section{Case Report}

A 30 year old female patient reported to the department of
Prosthodontics, with the chief complaints of inability in moving the neck, associated with tilting of head to the left side. Patient's family gave a history that she was normal at birth and till the age of 3 months. However, around 3 months of her age, they noticed that there was difficult in moving her head which had gradually increased to present level.

On extra oral examination, an obvious facial asymmetry was noted with the head tilted towards the left shoulder There was lack of facial fullness on the left side and an associated ocular dystopia. It was also noted that the left shoulder was not in level with the right shoulder and was raised above it (fig.01).Incidentally, intraoral examination revealed a cant in the occlusal plane, with left side at a lower level than the right side.

On radiographic examination of the cervical spine, it was 
noticed that there was a tilt to the left the vertebral column was straight with a bent in the neckspine. (fig.02)

Based on the above, the condition was diagnosed as Congenital M uscular Torticollis (wry neck) of the left side. A multidisciplinary protocol for treatment was planned involving the specialties of Prosthodontics, Oral surgery and Physiotherapy, to rehabilitate the patient. After obtaining the consent of the patient, pre-surgical impressions of both sides of the neck were obtained, surgery was carried out, followed by fitting of a stabilizing appliance fabricated in heat polymerized acrylic resin and physiotherapy neck exercises.

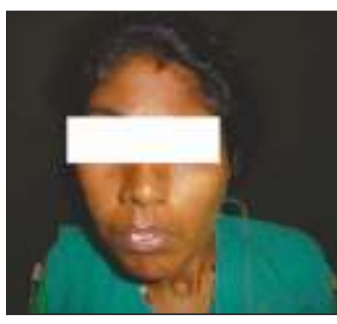

Fig. 01 : Pretreatment photograph

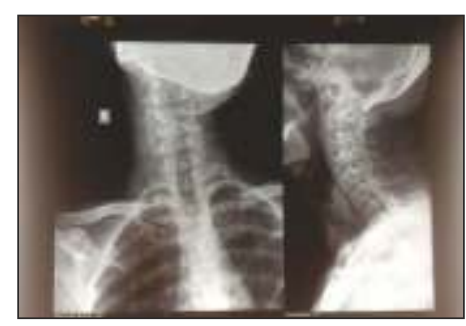

Fig.02 : Cervical spine $x$-ray AP/ LATERAL

\section{Fabrication of Stabilization and Activation Appliance Impressions\& working casts}

Prior to the surgery, impressions (Neocolloid, Zhermack, Italy) reinforced with type II gypsum (Kaldent, Kalabhai, India) were made of the affected side and the non-affected side of the neck and mandible (fig.03). Working casts were poured in type III gypsum product (Kalstone, Kalabhai, India) (Fig.04).

\section{Fabrication of the appliance}

Wax patterns were carved and a custom-made, functional, neck-stabilizing appliance was fabricated in the following manner using heat polymerizing acrylic resin.

The proposed appliance was made up of two braces- a shoulder brace and a mandibular brace with hooks on both the sides attached for the passage of a velcro strap band. The shoulder brace was long extending from scapula to clavicular region while the mandibular brace was extending from angle of mandible to the tip of the chin on both the sides. The appliance consists of a square shaped aluminium screw with a central hole to be used for the activation and deactivation. This would provide traction with each turn causing a movement of $2 \mathrm{~mm}$ (fig.05). The screw assembly was given on both the sides to provide stability to the head and neck position. The length of the screw was $100 \mathrm{~mm}$ on the unaffected and $75 \mathrm{~mm}$ on the affected side. Entire assembly was attached with a velcro strap band running from the hooks of mandibular braces on both sides crossing the midline of the sternum on clavicular area supporting the back of the patient (fig.09). The weight of the aluminium screws for activation of the muscles was kept minimal considering the comfort of the patient.

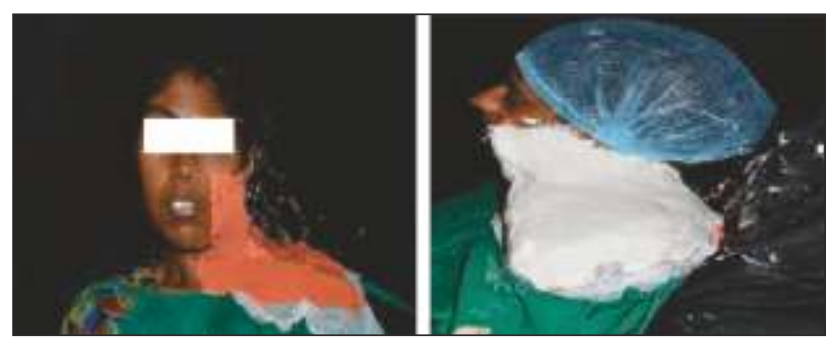

Fig.03 : Impression supported by dental plaster

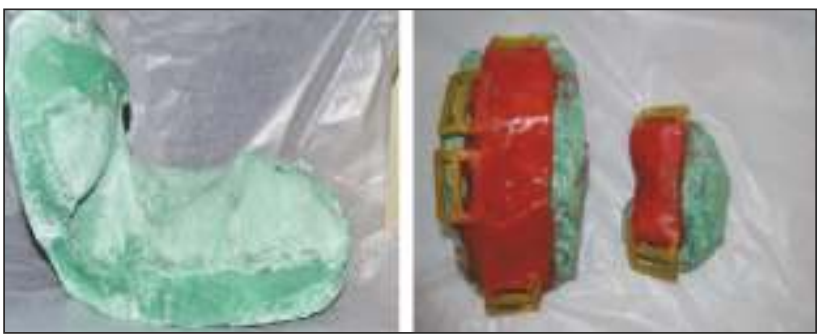

Fig. 04 : Stone cast and wax patterns
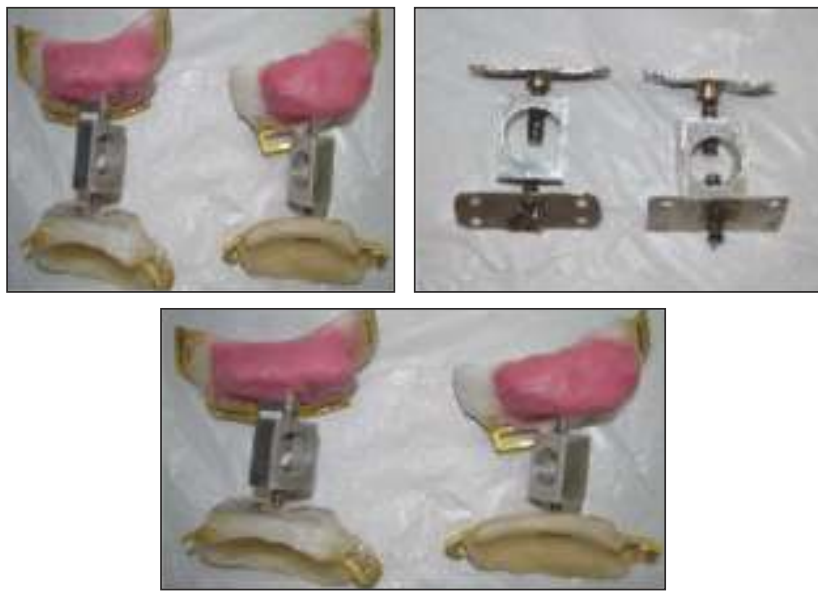

Fig. 05 : Stabilizing and activation appliance 


\section{Surgical Procedure}

An incision was placed $1 \mathrm{~cm}$ above the left side clavicle over the sternocleidomastoid muscle region involving the skin and subcutaneous tissue (fig. 06). Platysma muscle was identified, sectioned and reflected (fig.07). Sternocleidomastoid muscle was excised $2 \mathrm{~cm}$ above its head. Hemostasis was achieved and wound closure was done in two layers (fig.08). This surgical procedure was done with the intention of bipolar release of the sternocleidomastoid muscle.

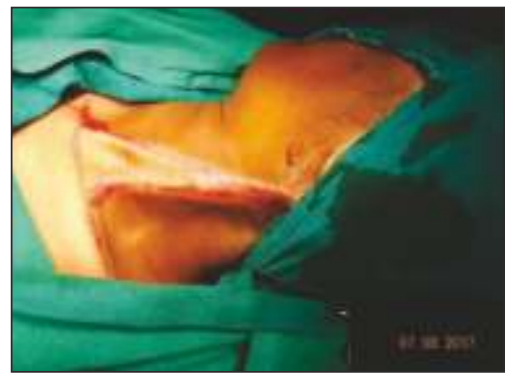

Fig.06 : Planned surgery

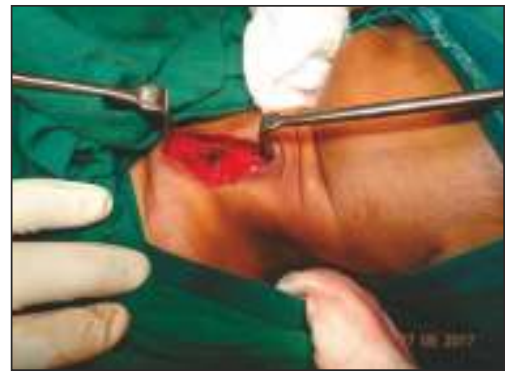

Fig.07 : Incision placed above the clavicle

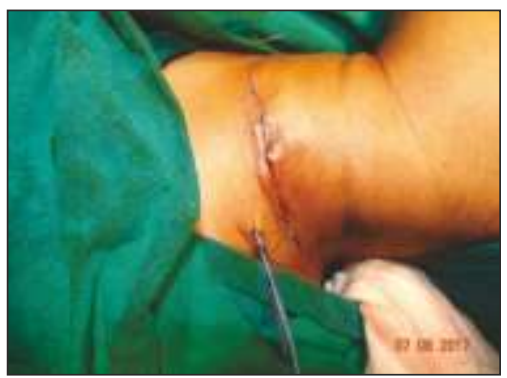

Fig. 08 : Surgical wound closure

The custom fabricated stabilizing $\&$ activation appliance was fitted to the patient 2 days after the surgery along with the application of the soft collar. Instructions were given on the method of fixing it and she was advised to wear it for 2 hours per day. Simple physiotherapy neck exercises involving stretching of Sternocleidomastoid muscle on the affected side were instituted along with the active appliance therapy (fig.09).

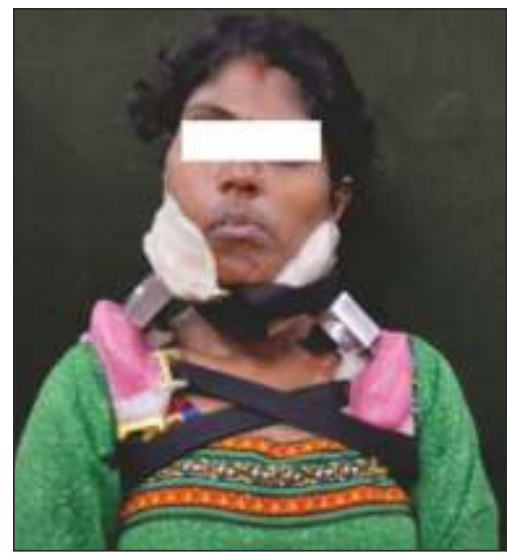

Fig.09 : Appliance fitted with the help of Velcro strap band

Patient was kept on a weekly follow up protocol for the planned activation of the appliance. The screws on the affected side were activated to the new position gradually increasing each time as the neck straightened up. This procedure was continued for three months to achieve the maximum and satisfactory head position in relation to the neck (Fig.10). After three months, the patient was able to rotate her neck satisfactorily and the head was positioned straight. However, for the correction of ocular dystopia and the occlusal cant of the dentition patient is advised to undergo further treatment.

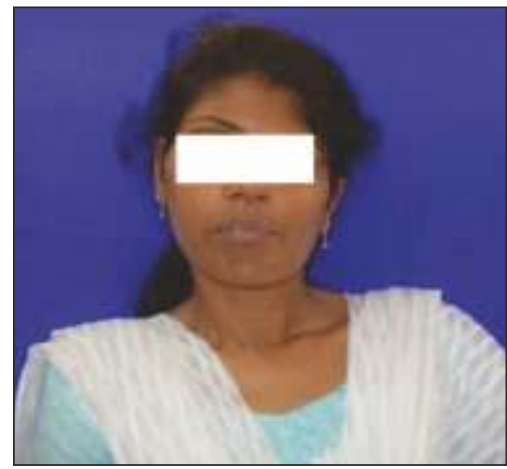

Fig. 10 : Post treatment

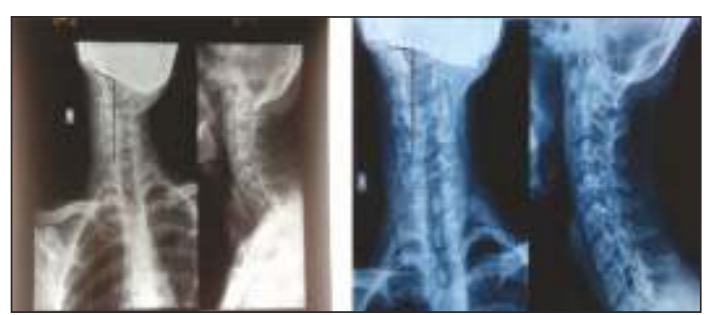

Fig. 11 : Comparison of pre and post cervical spine X-ray 


\section{Discussion}

Congenital muscular torticollis is a rare disorder where patient presents with a stiff sternocleidomastoid muscle, facial asymmetry, limited neck motions and elevation of ipsilateral shoulder. M ost often, this condition is associated with breech or difficult deliveries, but it may occur even in uncomplicated normal delivery or Cesarean section. The basic abnormality in this condition is the endomysial fibrosis with deposition of collagen and migration of fibroblasts around individual muscle fiber.

Bipolar release of sternocleidomastoid muscle is normally used in older children with severe deformity without the loss of neck column but it is difficult to estimate the extent of lengthening intraoperatively. ${ }^{3,4,5}$ In the present scenario, patient was 30 years old and hence surgical modality was opted to release the muscle followed by consolidation with custom made appliance therapy and physiotherapy.

Post surgically, the custom made stabilization and

\section{References}

1. Tatli B, Aydinli N, Çaliskan M, Ozmen M, Bilir F, Acar G. Congenital muscular torticollis: evaluation and classification. Pediatric neurology. 2006Jan 31;34(1):41-4.

2. Lee IJ, Lim SY, Song HS, Park M C. Complete tight fibrous band release and resection in congenital muscular torticollis. Journal of Plastic, Reconstructive \& Aesthetic Surgery. 2010 Jun 30;63(6):947-53.

3. Engin C, Yavuz SS, Sahin Fl. Congenital M uscular Torticollis: Is Heredity a Possible Factor in a Family with Five Torticollis Patients in Three Generations?. Plastic and reconstructive surgery. $1997 \mathrm{Apr}$ 1;99(4):1147-50

4. Wirth C], Hagena FW, Wuelker N, Siebert WE. Biterminaltenotomy for the treatment of congenital muscular torticollis. Long-term results. activation appliance was placed for 3 months, with a 2 hours application a day. Physiotherapy exercises were prescribed to hold the muscle in its new position and to prevent relapse from scar formation. The appliance was activated stepwise to stretch the affected muscle to achieve adequate length to straighten the neck. With frequent recall and assessments, the patient was functionally and esthetically rehabilitated. However, the correction of the occlusal cant and the dystopia were postponed as per the patient's desire.

\section{Conclusion}

A 30 year old female patient affected with congenital muscular torticollis was rehabilitated functionally and esthetically with the help of surgery and a custom made stabilization and activation appliance. Adjunctive physiotherapy exercises ensured that the neck maintained its functional rotation without any scar tissue formation post surgically.

JBJS. $1992 \mathrm{M}$ ar 1;74(3):427-34.

5. Chen CE, Ko JY. Surgical treatment of muscular torticollis for patients above 6 years of age. Archives of orthopaedic and trauma surgery. 2000 Feb 8;120 (3):149-51.

6. Shetty M, Hegde C, Burman J, Prasad KD, Tandan P. Muscular torticollis-functional and esthetic rehabilitation with an indigenously designed neck stabilizing appliance. The Journal of Indian Prosthodontic Society. 2008 Jan 1;8(1):52.

7. Patwardhan S, Shyam AK, Sancheti P, Arora P, Nagda T, Naik P. Adult presentation of congenital muscular torticollis. J Bone Joint Surg Br. 2011 Jun 1;93 (6):828-32. 